\title{
Genotyping reveals a wide heterogeneity of Tropheryma whipplei
}

Wenjun Li, ${ }^{1}$ Florence Fenollar, ${ }^{1}$ Jean-Marc Rolain, ${ }^{1}$ Pierre-Edouard Fournier, ${ }^{1}$ Gerhard E. Feurle, ${ }^{2}$ Christian Müller, ${ }^{3}$ Verena Moos, ${ }^{2}$ Thomas Marth, ${ }^{4}$ Martin Altwegg, ${ }^{5}$ Romana C. Calligaris-Maibach, ${ }^{6}$ Thomas Schneider, ${ }^{7}$ Federico Biagi, ${ }^{8}$ Bernard La Scola ${ }^{1}$ and Didier Raoult ${ }^{1}$

Correspondence Didier Raoult didier.raoult@gmail.com

Received 30 July 2007 Revised 25 October 2007 Accepted 25 October 2007

\author{
${ }^{1}$ Unité des Rickettsies, IFR 48, CNRS UMR 6020, Faculté de Médecine, Université de la \\ Méditerranée, 27 Boulevard Jean Moulin, 13385 Marseille Cedex 05, France \\ ${ }^{2}$ DRK Krankenhaus Neuwied, Germany \\ ${ }^{3}$ Universitätsklinik für Innere Medizin IV, Klinische Abteilung Gastroenterologie und Hepatologie, \\ Medizinische Universität, Wien, Austria \\ ${ }^{4}$ Department of Internal Medicine, St Josef Hospital, Zel, Germany \\ ${ }^{5}$ Bio-Analytica AG, Maihofstrasse 95a, 6000 Luzern 6, Switzerland \\ ${ }^{6}$ Department of Medical Microbiology, University of Zurich, Zurich, Switzerland \\ ${ }^{7}$ Department of Infectious Diseases, Charité, Campus Benjamin Franklin, Berlin, Germany \\ ${ }^{8}$ Gastroenterology Unit, Fondazione IRCCS, Policlinico San Matteo, University of Pavia, Italy
}

Tropheryma whipplei, the causative agent of Whipple's disease, is associated with various clinical manifestations as well as an asymptomatic carrier status, and it exhibits genetic heterogeneity. However, relationships that may exist between environmental and clinical strains are unknown. Herein, we developed an efficient genotyping system based on four highly variable genomic sequences (HVGSs) selected on the basis of genome comparison. We analysed 39 samples from 39 patients with Whipple's disease and 10 samples from 10 asymptomatic carriers. Twenty-six classic gastrointestinal Whipple's disease associated with additional manifestations, six relapses of classic Whipple's disease (three gastrointestinal and three neurological relapses), and seven isolated infections due to T. whipplei without digestive involvement (five endocarditis, one spondylodiscitis and one neurological infection) were included in the study. We identified 24 HVGS genotypes among 39 T. whipplei DNA samples from the patients and 10 T. whipplei DNA samples from the asymptomatic carriers. No significant correlation between HVGS genotypes and clinical manifestations of Whipple's disease, or asymptomatic carriers, was found for the 49 samples tested. Our observations revealed a high genetic diversity of $T$. whipplei strains that is apparently independent of geographical distribution and unrelated to bacterial pathogenicity. Genotyping in Whipple's disease may, however, be useful in epidemiological studies.

\footnotetext{
Abbreviations: HVGS, highly variable genomic sequence; ITS, internal transcribed spacer; UPGMA, unweighted pair group method with arithmetic mean.

The GenBank/EMBL/DDBJ accession numbers for the HVGS marker sequences of $T$. whipplei are given in Table 1.

A table showing a summary of clinical manifestations of Whipple's disease patients and asymptomatic carriers together with $T$. whipplei genotypes, and dendrograms showing the phylogenetic organization of the 24 HVGS genotypes constructed using the neighbour-joining and parsimony methods, are available as supplementary data with the online version of this paper.
}

\section{INTRODUCTION}

Infection with Tropheryma whipplei causes a variety of clinical manifestations, such as classic Whipple's disease marked by histological lesions in the gastrointestinal tract associated with diarrhoea, lymphadenopathy, arthritis, blood culture-negative endocarditis and isolated neurological infection without these typical histological lesions (Dutly \& Altwegg, 2001; Fenollar et al., 2007a; Fenollar \& Raoult, 2001; Marth \& Raoult, 2003). In addition, T. whipplei DNA has been detected in the environment and in 
asymptomatic carriers (Dutly et al., 2000; Ehrbar et al., 1999; Maiwald et al., 1998; Street et al., 1999). However, the prevalence of $T$. whipplei in the environment is still controversial because cultivation of $T$. whipplei directly from environmental samples is extremely difficult. The recent culture of T. whipplei from stools suggests a faecooral transmission of the disease (Raoult et al., 2006).

Several questions remain about the pathogenicity of $T$. whipplei. In theory, some strains may exhibit specific pathogenic virulence leading to classic Whipple's disease, others may cause endocarditis or central nervous system disease, and other strains may be apathogenic. Finally, it is not clear whether a differential geographical distribution exists among T. whipplei strains.

Completion of the genome sequences for two T. whipplei strains, TW08/27 and Twist, may allow a rational selection of appropriate genomic sequences for typing this bacterium (Bentley et al., 2003; Raoult et al., 2003). In this study, we selected four highly variable genomic sequences (HVGSs) in the aligned genome sequences of strains TW08/27 and Twist. Indeed, we have previously demonstrated that the greatest discriminatory power resides in the most variable sequence fragments between two closely related bacterial genomes (two strains of a species or two closely related species) as genotyping targets. Such a strategy has been successful for Rickettsia species (Fournier et al., 2004; Zhu et al., 2005), Bartonella species (Foucault et al., 2005; Li et al., 2006) and Yersinia pestis (Drancourt et al., 2004). The four HVGSs were used to evaluate the genetic diversity of $49 \mathrm{~T}$. whipplei strains detected in 39 patients with Whipple's disease and 10 asymptomatic carriers.

\section{METHODS}

Patients. Forty-nine samples were analysed in this study, including 39 specimens taken from 39 patients with a diagnosis of Whipple's disease on the basis of clinical manifestations, histological analysis and PCR assays, as previously reported (Fenollar et al., 2002, 2004, 2007a; Lepidi et al., 2003). The presentations linked to T. whipplei infections were defined as gastrointestinal or classic Whipple's disease characterized by positive periodic acid-Schiff (PAS)-staining lesions on small-bowel biopsies associated with additional manifestations, and as isolated infections characterized by the lack of the histological lesions on small-bowel biopsies, such as blood culture-negative endocarditis, isolated spondylodiscitis or isolated neurological infection. Relapses of classic Whipple's disease were divided into gastrointestinal and neurological relapses, depending on the clinical manifestations and the involved organs. Ten samples from 10 asymptomatic carriers were also included, corresponding to healthy people or patients without clinical manifestations of Whipple's disease and/or an excluded diagnosis of Whipple's disease based on the analysis of small-bowel biopsies, as previously reported (Ehrbar $e t$ al., 1999; Fenollar et al., 2007b; Schoniger-Hekele et al., 2007).

Study design. Detection of T. whipplei in specimens was carried out using PCR targeting the 16S-23S rRNA internal transcribed spacer (ITS), the $r p o B$ gene and/or a repeated sequence, as described in previous studies (Drancourt et al., 2001; Fenollar et al., 2002, 2004). Each T. whipplei strain detected in a human specimen was given a unique code containing ordinal information, i.e. country where the sample was collected, clinical manifestation, and organ or tissue from which the specimen was taken (Fig. 1 and Supplementary Table S1). For example, FrDDb1 stands for one T. whipplei strain detected in the duodenal biopsy $(\mathrm{Db})$ of a French patient (Fr) with classic Whipple's disease $(\mathrm{D}=$ digestive $)$.

DNA preparation. Total genomic DNA was extracted from each specimen using the QIAamp Tissue kit (Qiagen), as recommended by the manufacturer.

Selection of HVGSs. Four fragments were selected by identifying the most variable sequence fragments between $T$. whipplei strains Twist and TW08/27. To this end, the BLAST2 program (http:// www.ncbi.nlm.nih.gov/blast/bl2seq/wblast2.cgi) was used to align the genomic sequences of T. whipplei strains Twist (Raoult et al., 2003) (GenBank accession no. AE014184) and TW08/27 (Bentley et al., 2003) (GenBank accession no. BX072543). Then, genomic sequences with low sequence homology, with sizes ranging from 300 to $500 \mathrm{bp}$ and flanked by conserved sequences, were selected and used as genotyping markers in this study.

Design of primers, PCR amplification and sequencing. Amplifications of the ITS, the $r p o B$ gene and/or repeated sequences were performed using the previously described conditions and the tws3f/tws4r (Fenollar et al., 2002), TWRPOB.F/TWRPOB.R (Drancourt et al., 2001) and 5303F/5303R primer pairs (Fenollar et al., 2004), respectively. Primers for amplifying and sequencing the four HVGSs were selected using the Primer 3.0 software (http:// frodo.wi.mit.edu/cgi-bin/primer3/primer3_www.cgi). All primers were obtained from Eurogentec and are shown in Table 1. PCR reactions were carried out in a PTC-200 automated thermal cycler (MJ Research). Five microlitres of $3 \mathrm{ng} \mu^{-1}$ of each DNA solution were amplified in a $25 \mu \mathrm{l}$ reaction mixture containing $50 \mathrm{pM}$ each primer; $200 \mu \mathrm{M}$ (each) dATP, dCTP, dGTP and dTTP (Invitrogen); 1.5 U Hotstar Taq DNA polymerase (Qiagen); $2.5 \mu \mathrm{l} 10 \times$ PCR buffer; and $1 \mu \mathrm{l} 25 \mathrm{mM} \mathrm{MgCl}$. The following conditions were used for amplification: initial heat activation at $95{ }^{\circ} \mathrm{C}$ for $15 \mathrm{~min}$ was followed by 40 cycles of denaturation for $30 \mathrm{~s}$ at $94{ }^{\circ} \mathrm{C}$, annealing for $30 \mathrm{~s}$ at $55^{\circ} \mathrm{C}$, and extension for $1 \mathrm{~min}$ at $72{ }^{\circ} \mathrm{C}$. Amplification was completed by holding the reaction mixture for $5 \mathrm{~min}$ at $72{ }^{\circ} \mathrm{C}$ to allow complete extension of the PCR products. PCR products were purified by using the MultiScreen PCR filter plate (Millipore), as recommended by the manufacturer. Amplicons were sequenced in both directions using BigDye 1.1 chemistry (Applied Biosystems) on an ABI 3130xl automated sequencer (Applied Biosystems), as recommended by the manufacturer. Sterile water was used as a negative control in each PCR assay. All sequences were checked twice in both directions to ensure the reliability of the typing method.

Sequence analysis and phylogenetic analysis. Nucleotide sequences were edited using the Autoassembler package (PerkinElmer). For each HVGS, a sequence type was defined as a sequence exhibiting unique mutation(s). HVGS genotypes were defined as unique combinations of the four HVGS types.

The genotypic distribution of T. whipplei strains associated with four clinical manifestations was analysed to evaluate the correlation between HVGS genotypes and distinct clinical manifestations of Whipple's disease.

Multiple alignment of sequences was carried out using the CLUSTAL W software (Thompson et al., 1994). For phylogenetic analysis, sequences of the four HVGSs were concatenated. Phylogenetic relationships among $T$. whipplei genotypes were inferred using the unweighted pair group method with arithmetic mean (UPGMA), neighbour-joining and maximum-parsimony methods within the MEGA 3.1 software (Kumar et al., 2001). All different HVGS sequence types were deposited in GenBank (Table 1). 


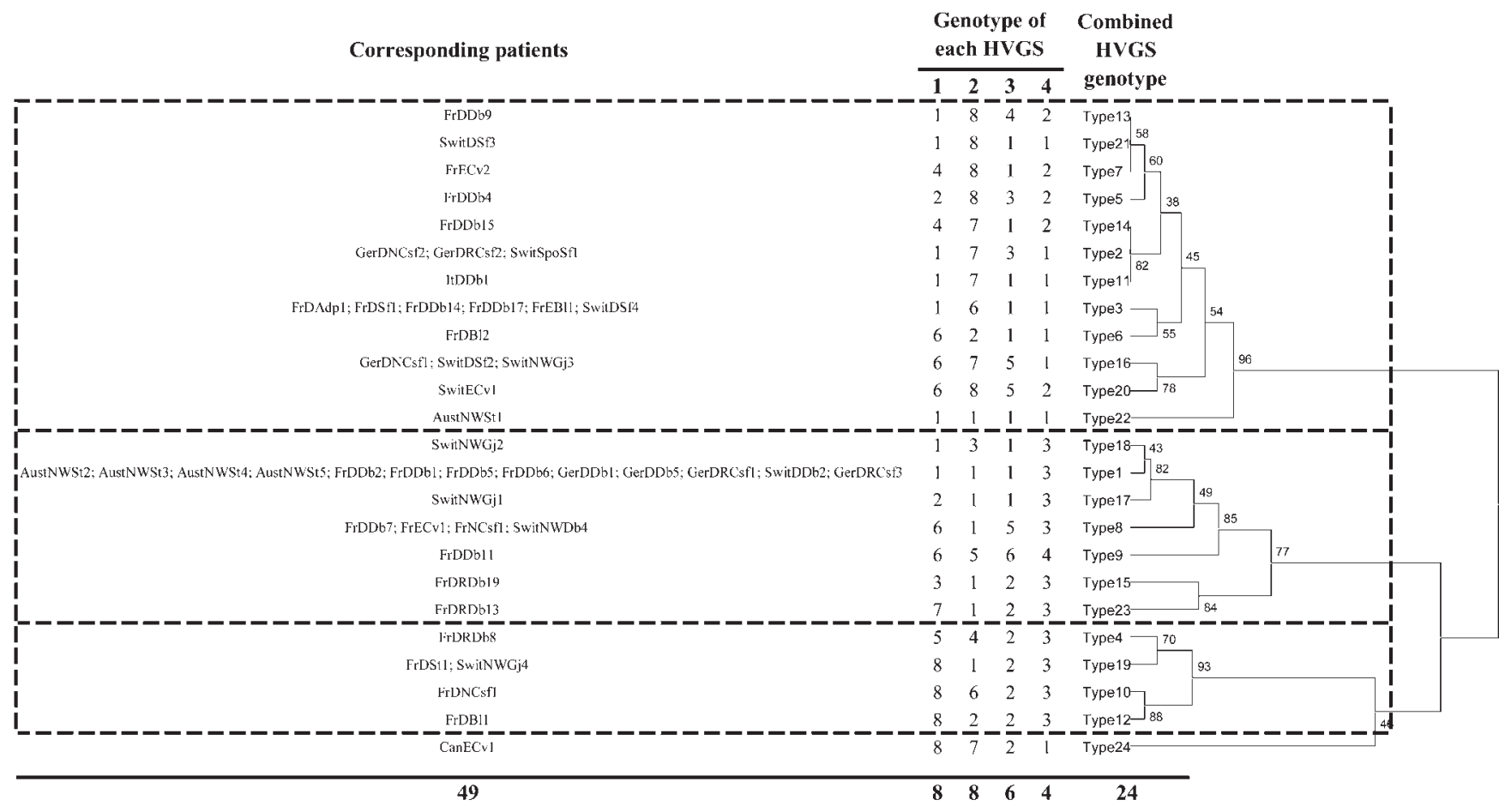

Fig. 1. Dendrogram showing the phylogenetic organization of the 24 HVGS genotypes, constructed using the UPGMA method. Sequences from the four HVGSs were concatenated. Bootstrap values are indicated at the nodes. Abbreviations: Fr, France; It, Italy; Ger, Germany; Can, Canada; Swit, Switzerland; Aust, Austria. D, digestive Whipple's disease; E, endocarditis due to T. whipplei; N, neurological Whipple's disease; Spo, spondylodiscitis; NW, without clinical manifestation of Whipple's disease; R, relapse. Db, duodenal biopsy; Cv,cardiac valve; Csf, cerebrospinal fluid; BI, blood; Adp, adenopathy; Sf, synovial fluid; Gj, gastric juice; St, stool.

\section{RESULTS}

\section{Patients}

The presentations linked to Whipple's disease were: 26 classic gastrointestinal Whipple's disease associated with additional manifestations; six relapses of classic Whipple's disease, including three gastrointestinal and three neurological relapses; and seven isolated infections due to $T$. whipplei, including five endocarditis, one spondylodiscitis and one neurological infection (Fig. 1, Supplementary Table S1). From the 10 patients without Whipple's disease, there were five stool samples taken from five Austrians, and four gastric juice samples and one duodenal biopsy taken from five Swiss (Fig. 1, Supplementary Table S1).

\section{Genotyping T. whipplei based on four HVGSs}

The four selected regions exhibited 92.51, 97.48, 93.42 and $89.22 \%$ identity between T. whipplei strains Twist and TW08/27, contained $14,8,10$ and 11 variable positions, and classified the 49 strains in $8,8,6$ and 4 sequence types, respectively (Tables 1 and 2).

By combining sequence types of the four sequences, the 49 tested T. whipplei strains were classified into 24 HVGS genotypes (Table 1). Of these, genotype 1, which was identified in 13 T. whipplei strains $(26.5 \%$ of the 49 tested T. whipplei DNA samples) was the most common in this study (Fig. 1). Genotypes 3, 8, 2, 16 and 19 included six, four, three, three and two T. whipplei DNA samples, respectively, which together accounted for $36.7 \%$ of the 49 tested T. whipplei strains (18/49) (Fig. 1). Each of the remaining 18 genotypes contained only one strain each (Fig. 1).

When stratifying genotypes according to clinical manifestations, 18 genotypes $(1-6,8-16,19,21$ and 23) were identified among the $32 \mathrm{~T}$. whipplei DNA samples detected in patients with classic Whipple's disease; five genotypes (3, 7, 8, 20 and 24) were identified among the five strains detected in patients with endocarditis; the single strain detected in a patient with spondylodiscitis was of genotype 2, which was also shared by another two strains from patients with classic Whipple's disease; the 10 strains detected in patients without Whipple's disease were classified into seven genotypes (1, 8, 16-19 and 22) (Fig. 1).

\section{Phylogenetic analysis of 24 HVGS genotypes}

Phylogenetic trees obtained from concatenation of the four HVGS sequences using the neighbour-joining and 
Table 1. Genomic information for the four HVGSs, primers used for amplification and sequencing of these HVGSs, and the reference numbers of the nucleotide sequences deposited in GenBank

Nucleotide sequences from the HVGS 1 marker were deposited in GenBank under reference numbers EF363910, EF363911, EF363912, EF363913, EF363914, EF363915, EF363916 and EF363917 for genotypes 1, 2, 3, 4, 5, 6, 7 and 8, respectively. Nucleotide sequences from the HVGS 2 marker were deposited in GenBank under reference numbers EF363917, EF363918, EF363919, EF363920, EF363921, EF363922, EF363923, EF363924 and EF363925 for genotypes 1, 2, 3, 4, 5, 6, 7 and 8, respectively. Nucleotide sequences from the HVGS 3 marker were deposited in GenBank under reference numbers EF363926, EF363927, EF363928, EF363929, EF363930 and EF363931 for genotypes 1, 2, 3, 4, 5 and 6, respectively. Nucleotide sequences from the HVGS 4 marker were deposited in GenBank under reference numbers EF363932, EF363933, EF363934 and EF363935 for genotypes 1, 2, 3 and 4, respectively.

\begin{tabular}{|c|c|c|c|c|c|c|c|c|}
\hline HVGS & $\begin{array}{l}\text { Content of } \\
\text { HVGS }\end{array}$ & Genotype & $\begin{array}{l}\text { Size } \\
(\mathbf{b p})\end{array}$ & $\begin{array}{c}\text { Position } \\
\text { on Twist } \\
\text { genome }\end{array}$ & $\begin{array}{c}\text { Position } \\
\text { on } \\
\text { TW08/27 } \\
\text { genome }\end{array}$ & $\begin{array}{c}\text { Identity } \\
\text { between } \\
\text { TW08/27 } \\
\text { and Twist }\end{array}$ & $\begin{array}{l}\text { Forward primer } \\
\text { sequence }\left(5^{\prime}-3^{\prime}\right)\end{array}$ & $\begin{array}{l}\text { Reverse primer } \\
\text { sequence }\left(5^{\prime}-3^{\prime}\right)\end{array}$ \\
\hline HVGS 1 & $\begin{array}{l}\text { TWT133 and } \\
\text { intergenic } \\
\text { spacer } \\
\text { (TWT133- } \\
\text { TWT134) }\end{array}$ & 8 & 227 & $\begin{array}{r}154935- \\
155161\end{array}$ & $\begin{array}{r}154333- \\
154553\end{array}$ & $92.51 \%$ & GCTGCGCGAAGTAATTTG & AGATACATGCGGAGATACT \\
\hline HVGS 2 & $\begin{array}{l}\text { proS/prolyl- } \\
\text { tRNA } \\
\text { synthetase } \\
\text { (TW183) }\end{array}$ & 8 & 318 & $\begin{array}{r}233956- \\
234273\end{array}$ & $\begin{array}{r}661022- \\
661339\end{array}$ & $97.48 \%$ & GCCTTGACTATGACATAATCAA & TCGGACTAAAAGTGCGACAC \\
\hline HVGS 3 & $\begin{array}{l}\text { secA-hp } \\
\text { (TWT131) } \\
\text { intergenic } \\
\text { spacer }\end{array}$ & 6 & 150 & $\begin{array}{r}152732- \\
152881\end{array}$ & $\begin{array}{r}152147- \\
152297\end{array}$ & $93.42 \%$ & TTTGTCATAGGCATTTCTGTAG & AGACCTCACTGTTATACGGAT \\
\hline HVGS 4 & $\begin{array}{l}\text { TW183 and } \\
\text { intergenic } \\
\text { spacer } \\
\text { (TWT183- } \\
\text { TWT184) }\end{array}$ & 4 & 162 & $\begin{array}{r}234449- \\
234610\end{array}$ & $\begin{array}{r}660680- \\
660846\end{array}$ & $89.22 \%$ & CGGATCTTCACGAAATGTCC & ATAACAAGAAGCTGGATATGC \\
\hline
\end{tabular}

maximum-parsimony methods showed similar phylogenetic classifications to the UPGMA method. The 24 HVGS genotypes were grouped into three clusters and one single branch linked with cluster 3 (Fig. 1, Supplementary Table S1). Cluster 1 contained 12 HVGS genotypes from $21 \mathrm{~T}$. whipplei strains, cluster 2 contained seven HVGS genotypes

Table 2. Polymorphism characteristics of the four HVGSs

The numbers in the HVGS polymorphism column show the position of each variable nucleotide with reference to the Twist strain. The allele before the number is that within the Twist strain and the alleles after the number are possible variable nucleotides within other T. whipplei strains. Inser, insertion; dele, deletion.

\begin{tabular}{|c|c|c|c|c|c|c|c|c|c|c|}
\hline \multirow{2}{*}{$\begin{array}{l}\text { HVGS } \\
\text { HVGS } 1\end{array}$} & \multicolumn{3}{|c|}{$\begin{array}{l}\text { Number of Number of } \\
\text { nucleotide genotypes } \\
\text { variations }\end{array}$} & \multicolumn{7}{|c|}{ HVGS polymorphism, with reference to the Twist strain } \\
\hline & 14 & 8 & $\begin{array}{l}\text { GGGGAT11-16dele } \\
\text { C124T }\end{array}$ & $\begin{array}{l}\text { G24T } \\
\text { G156A }\end{array}$ & $\begin{array}{l}\text { 48inserTA } \\
\text { G160A }\end{array}$ & $\begin{array}{l}\text { CT54-55TC } \\
\text { A166G }\end{array}$ & $\begin{array}{l}\text { G61A } \\
\text { G177T }\end{array}$ & $\begin{array}{l}\text { A68G } \\
\text { A223G }\end{array}$ & G89C & $\mathrm{C} 122 \mathrm{~T}$ \\
\hline HVGS 2 & 8 & 8 & C29T & T56C & $\mathrm{C} 140 \mathrm{~T}$ & A227G & A257G & A263G & G299A & G305A \\
\hline HVGS 3 & 10 & 6 & $\begin{array}{l}\text { G19dele } \\
\text { G103A }\end{array}$ & $\begin{array}{l}\text { A33G } \\
\text { A119G }\end{array}$ & T74AorG & $\mathrm{A} 75 \mathrm{~T}$ & G80A & G90A & 97inserCTorCC & $\mathrm{C} 99 \mathrm{~T}$ \\
\hline HVGS 4 & 11 & 4 & $\begin{array}{l}\text { T20C } \\
\text { C96T }\end{array}$ & $\begin{array}{l}\text { GT23-24AG } \\
\text { G146A }\end{array}$ & $\begin{array}{l}\text { TTT31-33GCC } \\
\text { G153T }\end{array}$ & 43inserGGGT & $\mathrm{C} 50 \mathrm{~T}$ & G60A & C69G & G83T \\
\hline $\begin{array}{l}\text { Total } \\
\text { (four } \\
\text { HVGSs) }\end{array}$ & 43 & 24 & & & & & & & & \\
\hline
\end{tabular}


from 23 T. whipplei strains, and cluster 3 contained four HVGS genotypes from five T. whipplei strains (Fig. 1). Genotype 24, identified in strain Twist and isolated from the cardiac valve of a Canadian patient with endocarditis, grouped with cluster 3 but with a low bootstrap value (Fig. 1). The phylogenetic classification of T. whipplei strains associated with distinct clinical manifestations was also analysed to evaluate the correlation between HVGS genotypes and distinct clinical forms (Fig. 1, Supplementary Table S1). Cluster 1 contained 14, three, one, one and two T. whipplei strains detected in patients with classic Whipple's disease, endocarditis, spondylodiscitis, neurological relapse, and patients without Whipple's disease, respectively (Fig. 1). Nine, one, one, two, two and seven strains detected in patients with classic Whipple's disease, neurological disorder, endocarditis, gastrointestinal relapse, neurological relapse, and individuals without Whipple's disease, respectively, were grouped into cluster 2 (Fig. 1). Cluster 3 contained three, one and one strains from patients with classic Whipple's disease, a patient with gastrointestinal relapse and a patient without Whipple's disease, respectively (Fig. 1). Thus, in this study, no significant correlation between HVGS genotypes and distinct manifestations of Whipple's disease was found on the basis of genotypic and phylogenetic analysis.

\section{DISCUSSION}

In this study, we found the combination of four HVGSs, selected following comparison of the complete genomes of two T. whipplei strains, useful for genotyping 49 clinical strains of this bacterium. Prior to our study, because of the fastidious nature of T. whipplei, several targets, such as $16 \mathrm{~S}$ rDNA, 23S rDNA, hsp65, atpD, tuf, groEL, rpoB and rnpD and 16S-23S rDNA ITS, had been studied to assess the phylogenetic organization of T. whipplei (Drancourt et al., 2001; Hinrikson et al., 1999b, 2000b; Maiwald et al., 1996, 2000, 2003; Morgenegg et al., 2000; Relman et al., 1992; Wilson et al., 1991). Most of these DNA sequences, however, are more conserved and reveal a limited genetic diversity among $T$. whipplei strains, although they contribute to some extent to genus and species classification. From sequences of domain III of $23 \mathrm{~S}$ rDNA, two sequence types were identified among $50 \mathrm{~T}$. whipplei strains detected in human specimens by Hinrikson et al. (2000a, b). Using a $620 \mathrm{bp}$ fragment of the $h s p 65$ gene, four genetic variants were identified among eight T. whipplei strains, but these strains had been selected from representative strains of three ITS genotypes (Morgenegg et al., 2000). ITS, currently the first-used molecular marker for typing $T$. whipplei infection, has so far allowed the differentiation of seven genotypes (Geissdorfer et al., 2001a; Hinrikson et al., 1999a, b; Maiwald et al., 2000). In a first study, ITS was found to be identical in nine Swiss strains (Hinrikson et al., 1999a). In a second study, three ITS sequence types were identified among 38 strains from 28 Swiss patients with Whipple's disease (Hinrikson et al., 1999b). Subsequently, five ITS sequence types were identified in 43 patients with Whipple's disease from the USA (11 patients), Germany (28 patients), Switzerland (three patients) and Austria (one patient) (Maiwald et al., 2000). More recently, a new ITS sequence type, type 7, was described in a German patient with endocarditis (Geissdorfer et al., 2001a). Combination of the four HVGSs allowed the identification of 24 HVGS genotypes among the 49 studied T. whipplei strains. The degree of sequence identity of HVGSs between strains Twist and TW08/27 (89.2-97.5\%) was lower than that of ITS (99.32\%) (Geissdorfer et al., 2001b; Hinrikson et al., 1999b). Thus, compared with previously described DNA sequence-based typing methods, our HVGS-based typing method was more discriminatory.

Recently, another typing strategy that combined three variable number of tandem repeats (VNTRs) and ITS discriminated 11 T. whipplei DNAs detected in patients with Whipple's disease. Each of the four markers (three VNTRs and ITS) identified two, three, six and two types among the $11 \mathrm{~T}$. whipplei DNAs, respectively (Maiwald et al., 2000). However, DNA size-based typing methods are not as discriminatory as sequence-based typing methods, because size variations may result from nucleotide insertions or deletions other than at VNTR sites. Phylogenetic analysis combining the two methods is difficult. In addition, the Maiwald et al. (2000) study was based on a limited number of strains.

Although the study of additional strains may be necessary, our results suggest that asymptomatic carriage and distinct clinical manifestations of Whipple's disease are not related to the genetic diversity of $T$. whipplei but rather to as yet unknown patient characteristics such as a deficient immune function, as proposed previously (Marth et al., 1994, 1997).

Although Whipple's disease is a rare systemic infectious disease, and mainly endemic in Europe and America, the relationships between genetic diversity and geographical distribution of the causative agent remain unclear. From ITS sequences, it appears that two common sequence types (types 1 and 2) are observed, with similar frequencies in patients from both the USA and Europe (Hinrikson et al., 1999b; Maiwald et al., 2000). Only one T. whipplei strain from outside Europe, Twist, was included in our study. Strain TW08/27, isolated from cerebrospinal fluid of a German patient with a neurological relapse of classic Whipple's disease, had the most frequent HVGS genotype, type 1 , and grouped into cluster 2 with 22 European $T$. whipplei strains (Fig. 1). Strain Twist, isolated from a cardiac valve of a Canadian patient with endocarditis due to T. whipplei, exhibited a specific HVGS genotype, type 24, and grouped with cluster 3 with an extremely low bootstrap value (Fig. 1). We could not draw any conclusion about the geographical distribution of $T$. whipplei. Ten HVGS genotypes were identified among 11 Swiss $T$. whipplei strains, in contrast to 15 , two and three HVGS genotypes identified among 25 French, five Austrian and 
seven German T. whipplei strains, respectively. The genetic diversity of Swiss T. whipplei strains seems to be higher than that observed in French, Austrian and German strains. However, more T. whipplei strains originating from different continents should be tested using this typing method to study the geographical distribution of $T$. whipplei. The high variability provides further evidence that positive PCR results in asymptomatic carriers are not, as was previously suggested (Dutly \& Altwegg, 2001; Fenollar et al., 2007a, b), due to carry-over contamination.

In conclusion, we identified four HVGSs based upon genomic comparison of T. whipplei strains TW08/27 and Twist. The four HVGSs classified 49 T. whipplei strains detected in patients with or without manifestations of Whipple's disease into 24 genotypes. However, no significant correlation between HVGS types and clinical manifestations of Whipple's disease or geographical distribution of T. whipplei was found. Our observations revealed a high genetic diversity of $T$. whipplei strains that apparently did not determine microbial pathogenicity and geographical distribution. Genotyping of T. whipplei may, however, be useful for epidemiological investigations such as the discrimination of recurrent Whipple's disease from reinfection with another strain.

\section{ACKNOWLEDGEMENTS}

We thank Melanie Ihrig for reviewing the manuscript. This project was funded by the 5th Framework Programme of the European Commission (ref. QLG1-CT-2002-01049).

\section{REFERENCES}

Bentley, S. D., Maiwald, M., Murphy, L. D., Pallen, M. J., Yeats, C. A., Dover, L. G., Nobertczak, H. T., Besra, G. S., Quail, M. A. \& other authors (2003). Sequencing and analysis of the genome of the Whipple's disease bacterium Tropheryma whipplei. Lancet 361, 637-644.

Drancourt, M., Carlioz, A. \& Raoult, D. (2001). $r p o B$ sequence analysis of cultured Tropheryma whipplei. J Clin Microbiol 39, 2425-2430.

Drancourt, M., Roux, V., Dang, L. V., Tran-Hung, L., Castex, D., Chenal-Francisque, V., Ogata, H., Fournier, P. E., Crubezy, E. \& Raoult, D. (2004). Genotyping, Orientalis-like Yersinia pestis, and plague pandemics. Emerg Infect Dis 10, 1585-1592.

Dutly, F. \& Altwegg, M. (2001). Whipple's disease and "Tropheryma whippelii'. Clin Microbiol Rev 14, 561-583.

Dutly, F., Hinrikson, H. P., Seidel, T., Morgenegg, S., Altwegg, M. \& Bauerfeind, P. (2000). Tropheryma whippelii DNA in saliva of patients without Whipple's disease. Infection 28, 219-222.

Ehrbar, H. U., Bauerfeind, P., Dutly, F., Koelz, H. R. \& Altwegg, M. (1999). PCR-positive tests for Tropheryma whippelii in patients without Whipple's disease. Lancet 353, 2214.

Fenollar, F. \& Raoult, D. (2001). Whipple's disease. Clin Diagn Lab Immunol 8, 1-8.

Fenollar, F., Fournier, P. E., Raoult, D., Gerolami, R., Lepidi, H. \& Poyart, C. (2002). Quantitative detection of Tropheryma whipplei DNA by real-time PCR. J Clin Microbiol 40, 1119-1120.
Fenollar, F., Fournier, P. E., Robert, C. \& Raoult, D. (2004). Use of genome selected repeated sequences increases the sensitivity of PCR detection of Tropheryma whipplei. J Clin Microbiol 42, 401-403.

Fenollar, F., Puéchal, X. \& Raoult, D. (2007a). Whipple's disease. $N$ Engl J Med 356, 55-66.

Fenollar, F., Trani, M., Davoust, B., Salle, B., Birg, M. L., Rolain, J. M. \& Raoult, D. (2007b). Carriage of Tropheryma whipplei in stools of sewer workers and human controls, but not in monkeys and apes. J Infect Dis (in press).

Foucault, C., La Scola, B., Lindroos, H., Andersson, S. G. \& Raoult, D. (2005). Multispacer typing technique for sequence-based typing of Bartonella quintana. J Clin Microbiol 43, 41-48.

Fournier, P. E., Zhu, Y., Ogata, H. \& Raoult, D. (2004). Use of highly variable intergenic spacer sequences for multispacer typing of Rickettsia conorii strains. J Clin Microbiol 42, 5757-5766.

Geissdorfer, W., Wittmann, I., Rollinghoff, M., Schoerner, C. \& Bogdan, C. (2001a). Detection of a new 16S-23S rRNA spacer sequence variant (type 7) of Tropheryma whippelii in a patient with prosthetic aortic valve endocarditis. Eur J Clin Microbiol Infect Dis 20, 762-763.

Geissdorfer, W., Wittmann, I., Seitz, G., Cesnjevar, R., Rollinghoff, M., Schoerner, C. \& Bogdan, C. (2001b). A case of aortic valve disease associated with Tropheryma whippelii infection in the absence of other signs of Whipple's disease. Infection 29, 44-47.

Hinrikson, H. P., Dutly, F. \& Altwegg, M. (1999a). Homogeneity of 16S-23S ribosomal intergenic spacer regions of Tropheryma whippelii in Swiss patients with Whipple's disease. J Clin Microbiol 37, 152-156.

Hinrikson, H. P., Dutly, F., Nair, S. \& Altwegg, M. (1999b). Detection of three different types of "Tropheryma whippelii" directly from clinical specimens by sequencing, single-strand conformation polymorphism (SSCP) analysis and type-specific PCR of their 16S-23S ribosomal intergenic spacer region. Int J Syst Bacteriol 49, 1701-1706.

Hinrikson, H. P., Dutly, F. \& Altwegg, M. (2000a). Analysis of the actinobacterial insertion in domain III of the 23S rRNA gene of uncultured variants of the bacterium associated with Whipple's disease using broad-range and "Tropheryma whippelii"-specific PCR. Int J Syst Evol Microbiol 50, 1007-1011.

Hinrikson, H. P., Dutly, F. \& Altwegg, M. (2000b). Evaluation of a specific nested PCR targeting domain III of the 23S rRNA gene of "Tropheryma whippelii" and proposal of a classification system for its molecular variants. J Clin Microbiol 38, 595-599.

Kumar, S., Tamura, K., Jakobsen, I. B. \& Nei, M. (2001). mega2: molecular evolutionary genetics analysis software. Bioinformatics 17, 1244-1245.

Lepidi, H., Fenollar, F., Gerolami, R., Mege, J. L., Bonzi, M. F., Chappuis, M., Sahel, J. \& Raoult, D. (2003). Whipple's disease: immunospecific and quantitative immunohistochemical study of intestinal biopsy specimens. Hum Pathol 34, 589-596.

Li, W., Chomel, B. B., Maruyama, S., Guptil, L., Sander, A., Raoult, D. \& Fournier, P. E. (2006). Multispacer typing to study the genotypic distribution of Bartonella henselae populations. J Clin Microbiol 44, 2499-2506.

Maiwald, M., Ditton, H. J., von Herbay, A., Rainey, F. A. \& Stackebrandt, E. (1996). Reassessment of the phylogenetic position of the bacterium associated with Whipple's disease and determination of the 16S-23S ribosomal intergenic spacer sequence. Int J Syst Bacteriol 46, 1078-1082.

Maiwald, M., Schuhmacher, F., Ditton, H. J. \& von Herbay, A. (1998). Environmental occurrence of the Whipple's disease bacterium (Tropheryma whippelii). Appl Environ Microbiol 64, 760-762. 
Maiwald, M., von Herbay, A., Lepp, P. W. \& Relman, D. A. (2000). Organization, structure, and variability of the rRNA operon of the Whipple's disease bacterium (Tropheryma whippelii). J Bacteriol 182, 3292-3297.

Maiwald, M., Lepp, P. W. \& Relman, D. A. (2003). Analysis of conserved non-rRNA genes of Tropheryma whipplei. Syst Appl Microbiol 26, 3-12.

Marth, T. \& Raoult, D. (2003). Whipple's disease. Lancet 361, 239-246. Marth, T., Roux, A., von Herbay, A., Meuer, S. C. \& Feurle, G. E. (1994). Persistent reduction of complement receptor $3 \alpha$-chain expressing mononuclear blood cells and transient inhibitory serum factors in Whipple's disease. Clin Immunol Immunopathol 72, 217-226.

Marth, T., Neurath, M., Cuccherini, B. \& Strober, W. (1997). Defects of monocyte interleukin 12 production and humoral immunity in Whipple's disease. Gastroenterology 113, 442-448.

Morgenegg, S., Dutly, F. \& Altwegg, M. (2000). Cloning and sequencing of a part of the heat shock protein 65 (hsp65) gene of Tropheryma whippelii and its use for the detection of Tropheryma whippelii in clinical specimens by PCR. J Clin Microbiol 38, 2248-2253.

Raoult, D., Ogata, H., Audic, S., Robert, C., Suhre, K., Drancourt, M. \& Claverie, J. (2003). Tropheryma whipplei Twist: a human pathogenic Actinobacteria with a reduced genome. Genome Res 13, 1800-1809.
Raoult, D., Fenollar, F. \& Birg, M. L. (2006). Culture of Tropheryma whipplei from the stool of a patient with Whipple's disease. $N$ Engl J Med 355, 1503-1505.

Relman, D. A., Schmidt, T. M., MacDermott, R. P. \& Falkow, S. (1992). Identification of the uncultured bacillus of Whipple's disease. N Engl J Med 327, 293-301.

Schoniger-Hekele, M., Petermann, D., Weber, B. \& Müller, C. (2007). Tropheryma whipplei in the environment - survey of sewage plant influxes and sewage plant workers. Appl Environ Microbiol 73, 2033-2035.

Street, S., Donoghue, H. D. \& Neild, G. H. (1999). Tropheryma whippelii DNA in saliva of healthy people. Lancet 354, 1178-1179.

Thompson, J. D., Higgins, D. G. \& Gibson, T. J. (1994). CLUSTAL W: improving the sensitivity of progressive multiple sequence alignment through sequence weighting, position-specific gap penalties and weight matrix choice. Nucleic Acids Res 22, 4673-4680.

Wilson, K. H., Blitchington, R., Frothingham, R. \& Wilson, J. A. (1991). Phylogeny of the Whipple's disease-associated bacterium. Lancet 338, 474-475.

Zhu, Y., Fournier, P. E., Ogata, H. \& Raoult, D. (2005). Multispacer typing of Rickettsia prowazekii enabling epidemiological studies of epidemic typhus. J Clin Microbiol 43, 4708-4712.

Edited by: K. E. Weaver 